\title{
The role of response selection and input monitoring in solving simple arithmetical products
}

\author{
MAUD DESCHUYTENEER and ANDRÉ VANDIERENDONCK \\ Ghent University, Ghent, Belgium
}

\begin{abstract}
Several studies have already shown that the central executive, as conceptualized in the working memory model of Baddeley and Hitch (1974), is important in simple mental arithmetic. Recently, attempts have been made to define more basic processes that underlie the "central executive." In this vein, monitoring, response selection, updating, mental shifting, and inhibition have been proposed as processes capturing executive control. Previous research has shown that secondary tasks that require a choice decision impair the calculation of simple sums, whereas input monitoring has not been found to be a sufficient condition to impair the calculation of sums (Deschuyteneer \& Vandierendonck, 2005). In the present article, we report data on the role of input monitoring and response selection in solving simple arithmetical products. In four experiments, participants solved one-digit products (e.g., $5 \times 7$ ) in a single-task, as well as in a dual-task, condition. Just as for solving simple sums, the results show a strong involvement of response selection in the calculating of simple products, whereas input monitoring does not seem to impair the calculation of such products. These findings give additional evidence that response selection may be one of the processes needed for solving simple mental arithmetic problems.
\end{abstract}

The multicomponent working memory model of Baddeley and Hitch (1974) has been frequently used by researchers as a framework for their investigation of the role of working memory in mental arithmetic (see DeStefano $\&$ LeFevre, 2004, for a review). In this framework, working memory is conceived of as a system that is responsible for the temporary storage and processing of information in a variety of cognitive tasks and consists of four components: the central executive and three slave systemsnamely, the phonological loop, the visuospatial sketchpad, and the episodic buffer (Baddeley, 1986, 1990, 2000; Baddeley \& Hitch, 1974). According to Baddeley and Hitch, the central executive is a modality-free limited-capacity attentional system that, among other things, controls the three slave systems during cognitive tasks. The phonological loop and the visuospatial sketchpad are responsible for the storage and the processing of verbal and visual/spatial information, respectively. The episodic buffer, which is a third, recently proposed slave system (Baddeley, 2000), is supposed to integrate information of the other two slave systems with information from episodic memory.

Most of the researchers who have studied the role of working memory in mental arithmetic have used the dualtask methodology, in which participants are required to perform two tasks concurrently (a primary and a second-

M.D. is a fellow (Grant 011D1201) of the Special Research Fund at Ghent University. The authors are indebted to Colin M. MacLeod, David C. Geary, and two anonymous reviewers for critical comments on an earlier version of this article. Correspondence concerning this article should be addressed to M. Deschuyteneer, Department of Experimental Psychology, Ghent University, Henri Dunantlaan 2, B-Ghent, Belgium (e-mail: maud .deschuyteneer@ugent.be or andre.vandierendonck@ugent.be). ary task). The general idea behind this method is that when performance on the primary task (e.g., an arithmetic task) is impaired while a secondary task that taxes specific cognitive functions is performed concurrently, it can be concluded that one or more of these cognitive functions are also involved in the primary task. A frequently replicated finding with this selective interference paradigm is that the central executive is involved in solving both simple and complex mental arithmetic problems (De Rammelaere, Stuyven, \& Vandierendonck, 1999, 2001; De Rammelaere \& Vandierendonck, 2001; Fürst \& Hitch, 2000; Hitch, 1978; Lemaire, Abdi, \& Fayol, 1996; Logie, Gilhooly, \& Wynn, 1994; Noël, Désert, Aubrun, \& Seron, 2001; Seitz \& Schumann-Hengsteler, 2000, 2002). The central executive is assumed to be responsible for controlling the calculations of the arithmetic problems.

Until now, the central executive has been conceptualized in the working memory model as a unitary system. There are, however, a number of indications that there are different components of executive functioning, such as input and output monitoring, inhibition, response selection, and memory updating (Baddeley, 1996; Miyake et al., 2000; Vandierendonck, 2000a, 2000b). For this reason, the finding that the central executive is involved in mental arithmetic fails to specify which particular processes are needed for solving mental arithmetic problems. The secondary tasks used in these dual-task studies on mental arithmetic may achieve their effect by mediation of one or more executive processes. Because several cognitive operations are involved in these tasks and which ones are involved is not always clear, the previous research does not allow conclusions about which (executive) processes are responsible for the effects observed. 
Deschuyteneer and Vandierendonck (2005) made a first attempt at specification of the executive processes involved in mental arithmetic. In particular, they studied the role of two executive processes - namely, input monitoring and response selection-in the solving of simple mental arithmetical sums. Input monitoring refers to a controlled scanning of all kinds of incoming information for elements relevant to the currently performed tasks. It requires a sharing of selective attentional resources between the active tasks. In situations in which the stream of events is predictable, this process of monitoring the input for relevant cues is thought to be easier than in situations in which the input stream is rather less predictable (see Vandierendonck, De Vooght, \& Van der Goten, 1998). Response selection is defined as a process that is required in order to assimilate the different response alternatives and to select a correct response between activated response alternatives (e.g., Schubert, 1999; Szmalec, Vandierendonck, $\&$ Kemps, 2005). Deschuyteneer and Vandierendonck reported four dual-task experiments in which participants had to solve simple sums in a control condition and in two dual-task conditions. In their first two experiments, they studied the role of temporal variation in input monitoring, whereas they explored the role of response selection in their last two experiments. To investigate the involvement of these two executive processes, they used secondary tasks that differed with respect to the presence of input monitoring and response selection, taking care not to put an important load on the other components specified by the working memory model. These tasks were a combination of two task characteristics (see Table 1). A first manipulation concerned the type of task. The task was either a simple reaction time task, in which the participants had to press a key each time they heard a tone, or a two-choice reaction time task, in which the participants were required to respond appropriately to two discriminate tones (a high and a low tone). In a simple reaction time task, a response has to be emitted as soon as the stimulus is presented. The two-choice reaction time task requires the stimulus to be detected and to be identified before the correct response can be selected. By comparing the degree of primary task impairment due to the choice reaction time task with the degree of impairment due to the simple reaction time task, it is possible to estimate the effect of response selection. A solid body of evidence has shown that if a response choice is required, response selection occurs (e.g., Lien \& Proctor, 2002; Pashler, 1994), whereas only a detection response is required in the other task variation (see also Schubert, 1999). The second manipulation used by Deschuyteneer and Vandierendonck concerned the presentation schedule of the stimuli (tones). The tones either were presented at a fixed rate or followed a random schedule. The rationale behind this manipulation is that in a random presentation schedule, the process of input monitoring will be called for more intensively than in a fixed presentation schedule (Vandierendonck et al., 1998). Because the tones are presented randomly over time so as to be unpredictable, it is possible that a control process (input monitoring) must be kept active during the input sequence. When the tones
Table 1

Combinations of Presentation Schedule and Type of Task Levels in Each of the Four Experiments

\begin{tabular}{ccc}
\hline & \multicolumn{2}{c}{ Type of Task } \\
\cline { 2 - 3 } $\begin{array}{c}\text { Presentation } \\
\text { Schedule }\end{array}$ & $\begin{array}{c}\text { Simple } \\
\text { Reaction }\end{array}$ & $\begin{array}{c}\text { Two-Choice } \\
\text { Reaction }\end{array}$ \\
\hline Fixed & 1,3 & 2,3 \\
Random & 1,4 & 2,4 \\
\hline
\end{tabular}

are presented at a fixed rate, the occurrence of the tones is predictable, and it can be hypothesized that this results in a more easily controlled process that does not require a monitoring of the input to the same extent. By comparing performance under concurrent fixed stimulus presentation with performance under concurrent random stimulus presentation, the effect of input monitoring was studied.

The results of Deschuyteneer and Vandierendonck (2005) showed that the calculation of simple sums is impaired by the presence of response selection. They found, however, no significant effect of input monitoring. These results suggest that input monitoring is probably not involved in solving simple sums, whereas response selection seems to be important in solving such sums. According to these authors, the lack of an effect of input monitoring could be due to the way in which the arithmetic problems were presented. The sums were presented at a fixed slow rate and did not disappear until after the participant had responded. So there was no need for the participants to control a sequence of events, and this is probably why there was no effect of input monitoring. Response selection, however, seems to impair the calculation of simple sums. The authors argued that response selection may be required during the retrieval of the correct sum from longterm memory, but it could also be that nonarithmetical processes, such as the encoding of the arithmetic problem and the production of the answer, are impaired by response selection. Most of the evidence, however, is in favor of the view that response selection is involved in the retrieval of arithmetical sums. According to Ashcraft (1992), arithmetical facts are retrieved from an interrelated network of associative links in long-term memory (see also Collins \& Loftus, 1975; Dehaene \& Cohen, 1995; Galfano, Mazza, Angrilli, \& Umiltà, 2004; Galfano, Rusconi, \& Umiltà, 2003; Geary, Widaman, \& Little, 1986; LeFevre, Bisanz, \& Mrkonjic, 1988; Miller, Perlmutter, \& Keating, 1984; Rusconi, Galfano, Speriani, \& Umiltà, 2004; Thibodeau, LeFevre, \& Bisanz, 1996; Verguts \& Fias, 2005). The idea is that the presentation of two numbers of an arithmetic problem results in the automatic activation of the number nodes in the associative network. Activation then automatically spreads from the presented nodes along associative links to related number nodes, such as the sum or the product. Also, Campbell's interference model (Campbell, 1987, 1995; Campbell \& Graham, 1985) and Siegler's associative strength model (Siegler, 1988; Siegler \& Shrager, 1984) agree that arithmetical facts are stored in an interrelated memory representation and predict response competition. Deschuyteneer and Vandierendonck 
(2005) argued that after the automatic activation of associated responses, a deliberate choice of the response to be executed is required to complete the retrieval.

The notion that a response selection occurs after the automatic activation of possible responses is consistent with findings from other sources. For instance, Bunge, Hazeltine, Scanlon, Rosen, and Gabrieli (2002) found, in their brain-imaging study, a dissociable contribution of frontal and parietal cortices to response selection. The goal of their study was to distinguish between brain areas representing candidate responses and areas selecting between competing response alternatives. They found that the parietal cortex is involved in the activation and the maintenance of a representation of possible responses. In contrast, the prefrontal cortex is recruited when there is a need to select among competing response alternatives. This contribution of frontal and parietal cortices to response selection was also found in other brain-imaging studies, such as those of Schumacher, Elston, and D'Esposito (2003) and Jiang and Kanwisher (2003a, 2003b). All these findings are in line with previous brain-imaging studies on mental arithmetic, which have revealed the involvement of frontal and parietal areas in arithmetical fact retrieval (e.g., Chochon, Cohen, van de Moortele, \& Dehaene, 1999; Pesenti, Thioux, Seron, \& De Volder, 2000; Rickard et al., 2000). Also, Lien and Proctor (2002) distinguished a response activation process and a response selection process in their review of behavioral studies on compatibility effects.

The purpose of the present study was to further explore the executive processes involved in solving mental arithmetic problems - namely, to explore the involvement of the processes of input monitoring and response selection in the calculation of another type of arithmetic problem (i.e., simple arithmetical products). This additionally allowed us to examine the above-mentioned idea that response selection may be involved in retrieving the correct answer from longterm memory. If it is correct that simple sums are solved by automatically activating the possible responses and retrieving the correct answer from these activated responses, it may be expected that concurrent response selection will also impair the calculation of simple multiplications. After all, more than simple sums, simple multiplications are assumed to be solved by retrieving the answers from longterm memory (e.g., Ashcraft, 1992; Campbell, 1987, 1995; Campbell \& Graham, 1985; Collins \& Loftus, 1975; Dehaene \& Cohen, 1995; Galfano et al., 2004; Galfano et al., 2003; Geary et al., 1986; LeFevre et al., 1988; Miller et al., 1984; Rusconi et al., 2004; Siegler, 1988; Siegler \& Shrager, 1984; Thibodeau et al., 1996; Verguts \& Fias, 2005).

Four dual-task experiments were performed, with the calculation of simple arithmetical products as the primary task. The secondary tasks were the same as those in the study of Deschuyteneer and Vandierendonck (2005). In Experiments 1 and 2, the effect of input monitoring was studied. This was done by comparing performance under concurrent fixed and random stimulus presentation conditions. In Experiment 1, the participants solved simple products in a single-task control condition and in dual-task conditions in which, concurrently with the products, a simple reaction time task (detection task) was performed either with fixed or with random stimulus presentation. Experiment 2 was completely similar, except that a two-choice reaction time task was used in the dual-task conditions - again, with a fixed or a randomly spaced stimulus presentation. In Experiments 3 and 4, the effect of response selection was studied by comparing performance under concurrent simple reaction and two-choice reaction conditions. In Experiment 3, the simple reaction time task and the two-choice reaction time task were combined with a fixed stimulus presentation. In Experiment 4, the two reaction time tasks were combined with a random stimulus presentation.

On the basis of the theoretical analysis, it was predicted that, just as for solving simple sums, the calculation of simple products would only be minimally (or not at all) affected by the variations in input monitoring. Just as for simple sums, there are no clear indications why the calculation of simple products calls on input monitoring. Due to the fact that the products are presented at a slow rate, there is no need for the participant to control a sequence of events. In contrast, if response selection is one of the executive processes involved in solving simple products, as was argued above, an impairment by response selection would be predicted.

\section{EXPERIMENT 1}

\section{Method}

Participants. Twenty first-year psychology students of Ghent University ( 9 of them female and 11 male) participated in the present experiment for course requirements and credit. The mean age was 19.4 years (range, $18.5-22.5$ years).

Stimuli. The stimuli consisted of 56 simple products presented in the form $a \times b$; the numbers and the symbol were separated by spaces equal to the width of one character. The terms $a$ and $b$ were always onedigit numbers from 2 to 9 . The numbers 0 and 1 were omitted because there is evidence that problems like $x \times 0$ and $x \times 1$ are solved by retrieving specific rules that guide their solution (see Ashcraft, 1982; Baroody, 1985). Also, problems composed of a repeated operand, the so-called ties (e.g., $4 \times 4$ ), were excluded. Research has shown that ties are typically solved much more quickly and more accurately than nontie problems (e.g., Blankenberger, 2001; Campbell, 1999b; Campbell \& Graham, 1985; Campbell \& Gunter, 2002).

Procedure and Design. The participants were tested individually in a quiet room. They were seated at a Pentium III PC with a 15 -in. color monitor. The participants were instructed to solve the problems as accurately and as quickly as possible by pronouncing the answer into a voice key microphone that was connected to the game port of the computer. The latencies were measured by means of the voice key.

Each trial consisted of the following steps. First, a fixation mask (!!!!!) was presented for $500 \mathrm{msec}$ in the middle of the computer screen. Next, the stimuli were presented horizontally in the center of the computer screen. Both the fixation point and the stimuli were presented in white on a black background. The arithmetic problem remained visible until the participant responded or until $10 \mathrm{sec}$ had passed without an answer. When the voice key was triggered, the problem disappeared, and a black screen was presented. After the arithmetic problem disappeared, the experimenter typed the participant's answer on the computer keyboard and struck the enter key. Immediately thereafter, the next trial appeared.

There were two dual-task conditions: one based on a fixed sequence of simple reaction time (SRT-F) tasks and another based on a random schedule of simple reaction time (SRT-R) tasks. In the SRT-F condition, the participants were required, while solving the products, to hit 
the zero key on the numeric pad with their right index finger as quickly as possible each time they heard a tone. Each tone had a frequency of $262 \mathrm{~Hz}$ and lasted for $200 \mathrm{msec}$. The rate at which the tones were presented was regular. The interval between two tones was 1,700 msec. Also, in the SRT-R condition, the participants were required, while solving the products, to hit the zero key on the numeric pad with the right index finger as quickly as possible after each tone. The difference from the SRT-F condition was that the intervals between the tones varied randomly. In this condition, the interval between two tones was either 900 or $1,500 \mathrm{msec}$. There were also three single-task conditions: a control condition in which the participants solved the products without a secondary task, a second condition in which the SRT-F task had to be performed alone, and a third condition in which the SRT-R task had to be performed alone. All the participants were randomly assigned to one of the counterbalanced orders (on the basis of a Latin square design) of the five within-subjects conditions.

Before the actual experiment began, the participants read the instructions and solved 10 random practice trials in order to become familiarized with the apparatus, the procedure, and the stimulus display. The 56 stimuli were presented four times each in the control condition and the two dual-task conditions, so that the participants had to solve 224 simple products in each of these conditions. Thus, all the participants solved exactly 682 simple products $[10$ practice trials + $(224 \times 3)$ experimental trials]. The presentation of all the problems was randomized within the conditions.

\section{Results}

In all the experiments in the present study, the data of the arithmetic task were acquired in repeated measures designs and were analyzed on the basis of the multivariate general linear model. Performance was measured in terms of latencies (in milliseconds) and accuracy (i.e., proportions of correct answers). Also, the effect sizes are reported. For reporting the effect sizes, we followed Cohen's (1988) recommendation and used Cohen's $d$ as the measure of effect size. Performance on the secondary tasks was measured by means of the average reaction time to the tones, the standard deviations on the reaction times, and the number of anticipatory errors per second. For the performance on the secondary tasks under the random presentation schedule, the correlation of intertone and intertap intervals also was taken into account; for the performance on the two-choice reaction time tasks, the number of response selection errors per second was used as an additional measure. But most important, in each dual-task condition of all four of the experiments, correlations were calculated between each of the secondary tasks separately and between primary (arithmetic) task performance to investigate whether there were trade-offs between primary and secondary task performance.

Latencies. Of the 13,440 experimental trials (672 products solved by 20 participants), 1,217 , or $9.1 \%$, were dropped due to coughing or voice key failure. These eliminated trials were evenly distributed among the three conditions. Only the latencies for the correctly solved products were included in the analysis. A multivariate analysis based on the general linear model was conducted on the variable of load (three levels: control, SRT-F, or SRT-R). Planned comparisons showed that the difference between the control condition $(M=1,066 \mathrm{msec})$ and the SRT-F condition $(M=$ $1,149 \mathrm{msec})$ was not significant $\left[F(1,19)=3.40, M S_{\mathrm{e}}=\right.$ $20,084.13, p=.08 ; d=.25]$; there was no significant dualtask coordination cost. Neither was the difference between the SRT-F and the SRT-R $(M=1,142 \mathrm{msec})$ conditions significant $\left(F<1, M S_{\mathrm{e}}=18,284.96 ; d=.02\right)$.

In a second analysis, the problem size $\times$ load interaction was investigated by making a distinction between small and large problems. The stimuli were divided into small problems (with products smaller than or equal to 25) and large problems (with correct products larger than 25). This distinction was based on Campbell (1997; Campbell, Kanz, \& Xue, 1999). Mean latencies and standard deviations are displayed in Table 2 . The data were analyzed by means of a 3 (load: control, SRT-F, or SRT-R) $\times 2$ (problem size: small or large) within-subjects design. The main effect of problem size $\left[F(1,19)=36.31, M S_{\mathrm{e}}=176,068, p<.001\right]$ was significant, but the main effect of load $\left[F(2,38)=1.57, M S_{\mathrm{e}}=\right.$ $57,377, p=.22]$ and the load $\times$ problem size interaction $\left(F<1, M S_{\mathrm{e}}=19,630\right)$ were not.

Accuracy. The accuracy was high: $M=.96$ in the control condition, $M=.95$ in the SRT-F condition, and $M=$ .96 in the SRT-R condition. There were no significant differences. However, due to the fact that the accuracy was high, these data are not very sensitive, and differences in accuracy must be interpreted with caution. Because the accuracy was high in all the experiments, this remark also applies to the other experiments.

An error analysis also was conducted, and this analysis showed that $90 \%$ of the errors were common table-related intrusions and that these intrusions were evenly distributed across conditions.

Secondary task performance. The data for the secondary tasks are displayed in Table 3. For each of these tasks, performance was worse in the dual-task condition than in the single-task condition $\left[F(1,19)=60.26, M S_{\mathrm{e}}=\right.$ $12,479, p<.001$, for the SRT-F task, and $F(1,19)=370.95$, $M S_{\mathrm{e}}=850, p<.001$, for the SRT-R task].

Correlations between arithmetic task performance and each of the secondary tasks separately were also calculated. It appeared that in the SRT-F condition, there was a positive correlation between primary task performance and SRT-F

Table 2

Experiments 1 and 2: Mean Latencies (With Standard Deviations, in Milliseconds) for Small and Large Arithmetic Problems

\begin{tabular}{|c|c|c|c|c|}
\hline \multirow[b]{3}{*}{ Condition } & \multicolumn{4}{|c|}{ Problem Size } \\
\hline & \multicolumn{2}{|c|}{ Small } & \multicolumn{2}{|c|}{ Large } \\
\hline & $M$ & $S D$ & $M$ & $S D$ \\
\hline \multicolumn{5}{|c|}{ Experiment 1} \\
\hline Control & 864 & 117 & 1,309 & 527 \\
\hline SRT-F & 942 & 192 & 1,407 & 539 \\
\hline SRT-R & 924 & 180 & 1,399 & 535 \\
\hline \multicolumn{5}{|c|}{ Experiment 2} \\
\hline Control & 993 & 209 & 1,491 & 426 \\
\hline CRT-F & 1,190 & 379 & 1,669 & 675 \\
\hline CRT-R & 1,287 & 467 & 1,776 & 756 \\
\hline
\end{tabular}

Note-SRT-F, fixed schedule of simple reaction time tasks; SRT-R, random schedule of simple reaction time tasks; CRT-F, fixed schedule of two-choice reaction time tasks; CRT-R, random schedule of two-choice reaction time tasks. 
Table 3

Experiment 1: Fixed Simple Reaction Time (SRT-F) and Random Simple Reaction Time (SRT-R) Tapping Performance Measures

\begin{tabular}{clcrccc}
\hline & & \multicolumn{2}{c}{ RT $(\mathrm{msec})$} & Anticipations/ & \\
\cline { 3 - 4 } Task & Condition & Average & $S D$ & Second & Correlation \\
\hline SRT-F & Single task & 281 & 98 & 0.02 & \\
& Dual task & 494 & 360 & 0.04 & \\
\multirow{2}{*}{ SRT-R } & Single task & 271 & 63 & 0.01 & .97 \\
& Dual task & 432 & 257 & 0.05 & .86 \\
\hline
\end{tabular}

task performance $(r=.49, p<.05)$. The correlation between primary task performance and performance on the SRT-R task in the SRT-R condition was not significant $(r=-.16, p=.49)$. Hence, there were no trade-offs between primary task and secondary task performance.

\section{Discussion}

In this experiment, the effect of input monitoring for solving simple products was studied. This was done by comparing two dual-task conditions. In the first dual-task condition, the products had to be solved concurrently with an SRT-F task. In the second dual-task condition, the secondary task was an SRT-R task. The results showed no significant difference between these two dual-task conditions. This supports the conclusion that there was no significant effect of input monitoring. The analyses also showed no significant load $\times$ problem size interaction. The possible implications of this finding will be elaborated in the General Discussion section.

In Experiment 2, we tested whether this result could be generalized to other task conditions. Performance on the primary task was compared under fixed two-choice reaction time and random two-choice reaction time conditions.

\section{EXPERIMENT 2}

\footnotetext{
Method

Participants. Twenty first-year psychology students of Ghent University ( 15 of them female and 5 male) participated in the present experiment for course requirements and credit. The mean age was 21.4 years (range, 18.3-43.3 years). None of them had participated in Experiment 1.

Stimuli and Procedure. The stimuli and the procedure were identical to those in Experiment 1. The only difference was in the secondary tasks used in the two dual-task conditions and in the corresponding single-task conditions. This experiment was based on the fixed two-choice reaction time task and the random two-choice reaction time task as secondary tasks. As in Experiment 1, there were five counterbalanced conditions based on a Latin square: two dualtask conditions and three single-task conditions. One condition was based on a fixed schedule of two-choice reaction time tasks (CRT-F). In this condition, the participants were required, while solving the products, to hit the 1 key or the 4 key on the numeric pad as quickly as possible each time they heard a low $(262-\mathrm{Hz})$ or a high $(524-\mathrm{Hz})$ tone, respectively. The rate at which the tones were presented was regular (every $1,700 \mathrm{msec}$ ). A second condition was based on a random sequence of two-choice reaction time tasks (CRT-R). This condition was completely similar, except that the intervals between the tones varied randomly. The interval between two tones was either 900 or $1,500 \mathrm{msec}$. In the control condition, the participants solved the
}

products without a secondary task. In a fourth condition, the CRT-F task had to be performed alone, and in a last condition, the CRT-R task had to be performed alone.

\section{Results}

Latencies. Of the 13,440 experimental trials $(672$ products solved by 20 participants), 1,201, or $8.9 \%$, were dropped due to coughing or voice key failure. These eliminated trials were evenly distributed among the conditions. Only the latencies of the correctly solved products were included in the analysis. A multivariate analysis based on the general linear model was conducted on the variable of load (three levels: control, CRT-F, or CRT-R). Planned comparisons showed that the difference between the control condition $(M=1,219 \mathrm{msec})$ and the CRT-F condition $(M=1,426 \mathrm{msec})$ was significant $\left[F(1,19)=4.84, M S_{\mathrm{e}}=\right.$ $88,546.3, p<.05 ; d=.50]$; there was a significant dualtask coordination cost. The difference between the CRT-F and the CRT-R $(M=1,507 \mathrm{msec})$ conditions was not significant $\left(F<1, M S_{\mathrm{e}}=134,454.2 ; d=.15\right)$.

As in Experiment 1 the problem size $\times$ load interaction was investigated by making a distinction between small and large problems. The data were analyzed by means of a 3 (load: control, CRT-F, or CRT-R) $\times 2$ (problem size: small or large) within-subjects design. Mean latencies and standard deviations are displayed in Table 2. There were significant main effects of load $\left[F(2,38)=3.85, M S_{\mathrm{e}}=223,865\right.$, $p<.05]$ and of problem size $\left[F(1,19)=65.33, M S_{\mathrm{e}}=\right.$ $109,520, p<.001]$. The load $\times$ problem size interaction was not significant $\left(F<1, M S_{\mathrm{e}}=19,125\right)$.

Accuracy. Accuracy was high: $M=.95$ in the control condition, $M=.95$ in the CRT-F condition, and $M=.94$ in the CRT-R condition. There were no significant differences.

In this experiment, an error analysis also was conducted. This analysis showed that $88 \%$ of the errors were common table-related intrusions and that these intrusions were evenly distributed across conditions.

Secondary task performance. The data for the secondary tasks are displayed in Table 4. For each of these tasks, dual-task performance was worse than single-task performance $\left[F(1,19)=409.90, M S_{\mathrm{e}}=1,124, p<.001\right.$, for the CRT-F task, and $F(1,19)=283.59, M S_{\mathrm{e}}=531, p<$ .001 , for the CRT-R task].

Correlations between arithmetic task performance and each of the secondary tasks were also calculated. It appeared that the correlation between primary task performance and each of the tasks in the dual-task condition was not significant $(r=.05, p=.82$, in the CRT-F condition, and $r=$ $.40, p=.08$, in the CRT-R condition). So, just as in the previous experiment, there were no trade-offs between primary task and secondary task performance.

\section{Discussion}

As in Experiment 1, the results showed no significant effect of input monitoring in the solving of simple products. There was no significant difference when performance was compared under concurrent CRT-F and CRT-R conditions. Taken together, it seems that input monitoring is not involved in solving simple products. The effect size 
Table 4

Experiment 2: Fixed Two-Choice Reaction Time (CRT-F) and

\begin{tabular}{|c|c|c|c|c|c|c|}
\hline \multirow[b]{2}{*}{ Task } & \multirow[b]{2}{*}{ Condition } & \multicolumn{2}{|c|}{ RT (msec) } & \multirow{2}{*}{$\begin{array}{l}\text { Anticipations/ } \\
\text { Second }\end{array}$} & \multirow{2}{*}{$\begin{array}{l}\text { Response Selection } \\
\text { Errors/Second }\end{array}$} & \multirow[b]{2}{*}{ Correlation } \\
\hline & & Average & $S D$ & & & \\
\hline \multirow[t]{2}{*}{ CRT-F } & Single task & 428 & 102 & 0.01 & 0.02 & \\
\hline & Dual task & 638 & 321 & 0.03 & 0.04 & \\
\hline \multirow[t]{2}{*}{ CRT-R } & Single task & 419 & 112 & 0.02 & 0.03 & .90 \\
\hline & Dual task & 538 & 268 & 0.05 & 0.09 & .90 \\
\hline
\end{tabular}

for the latencies was small, and as in Experiment 1, there was no significant load $\times$ problem size interaction.

\section{EXPERIMENT 3}

In the next two experiments, the role of response selection was investigated. In Experiment 3, performance on the primary task was compared under concurrent SRT-F and CRT-F conditions. In Experiment 4, the effect was studied by comparing two dual-task conditions in which the products had to be solved concurrently with an SRT-R and a CRT-R task.

\section{Method}

Participants. Twenty first-year psychology students (11 of them female and 9 male) at Ghent University participated in the present experiment for course requirements and credit. The mean age was 19.6 years (range, 18.4-24.5 years). None of them had participated in either of the previous experiments.

Stimuli and Procedure. The stimuli and the procedure were the same as those in Experiments 1 and 2. The only difference pertained to the secondary tasks used. This experiment was based on the SRT-F task (see Experiment 1) and the CRT-F task (see Experiment 2) as secondary tasks. These tasks were implemented in exactly the same way as in the previous experiments.

\section{Results}

Latencies. Of the 13,440 experimental trials ( 672 products solved by 20 participants), 1,359 , or $10.1 \%$, were dropped due to coughing or voice key failure. In this experiment also, the eliminated trials were evenly distributed among the conditions. Only the latencies of the correctly solved products were included in the analysis. A multivariate analysis based on the general linear model was conducted on the variable of load (three levels: control, SRT-F, or CRT-F). Planned comparisons showed that the difference between the control condition $(M=1,139 \mathrm{msec})$ and the SRT-F condition $(M=1,184 \mathrm{msec})$ was not significant $\left[F(1,19)=1.91, M S_{\mathrm{e}}=10,669.21, p=.18 ; d=.13\right]$. However, the difference between the SRT-F condition and the CRT-F condition $(M=1,280 \mathrm{msec})$ was significant $\left[F(1,19)=4.99, M S_{\mathrm{e}}=18,203.86, p<.05 ; d=.27\right]$; the products were solved more quickly in the SRT-F condition than in the CRT-F condition.

The problem size $\times$ load interaction was investigated by analyzing the data by means of a 3 (load: control, SRT-F, or CRT-F) $\times 2$ (problem size: small or large) within-subjects design. Mean latencies and standard deviations are displayed in Table 5. The main effects of load $[F(2,38)=7.55$, $\left.M S_{\mathrm{e}}=29,099, p<.01\right]$ and of problem size $[F(1,19)=$
39.77, $\left.M S_{\mathrm{e}}=166,356, p<.001\right]$ were significant. The load $\times$ problem size interaction was not significant $(F<1$, $\left.M S_{\mathrm{e}}=7,650\right)$.

Accuracy. The accuracy was high: $M=.97$ in the control condition, $M=.97$ in the SRT-F condition, and $M=.96$ in the CRT-F condition. There were no significant differences.

As in Experiments 1 and 2, an error analysis was conducted. This analysis showed that $90 \%$ of the errors were common table-related intrusions and that these intrusions were evenly distributed across conditions.

Secondary task performance. The data for the secondary tasks are displayed in Table 6. For each of these tasks, performance was worse in the dual-task condition than in the single-task condition $\left[F(1,19)=73.70, M S_{\mathrm{e}}=\right.$ $8,863, p<.001$, for the SRT-F task, and $F(1,19)=296.83$, $M S_{\mathrm{e}}=1,296, p<.001$, for the CRT-F task].

Also, in this experiment, correlations between arithmetic task performance and each of the secondary tasks were calculated separately. The correlation between primary task performance and SRT-F task performance in the SRT-F condition was not significant $(r=.10, p=.67)$, whereas there was a positive correlation in the CRT-F condition between primary task performance and performance on the CRT-F task $(r=.48, p<.05)$. Thus, there were no trade-offs between primary task and secondary task performance.

\section{Discussion}

The effect of response selection was investigated by comparing performance on the primary task in two dual-

Table 5

Experiments 3 and 4: Mean Latencies (With Standard Deviations, in Milliseconds) for Small and Large Arithmetic Problems

\begin{tabular}{|c|c|c|c|c|}
\hline \multirow[b]{3}{*}{ Condition } & \multicolumn{4}{|c|}{ Problem Size } \\
\hline & \multicolumn{2}{|c|}{ Small } & \multicolumn{2}{|c|}{ Large } \\
\hline & $M$ & $S D$ & $M$ & $S D$ \\
\hline \multicolumn{5}{|c|}{ Experiment 3} \\
\hline Control & 908 & 197 & 1,400 & 528 \\
\hline SRT-F & 965 & 196 & 1,412 & 475 \\
\hline CRT-F & 1,061 & 234 & 1,531 & 525 \\
\hline \multicolumn{5}{|c|}{ Experiment 4} \\
\hline Control & 919 & 162 & 1,381 & 306 \\
\hline SRT-R & 1,044 & 258 & 1,530 & 420 \\
\hline CRT-R & 1,187 & 294 & 1,639 & 465 \\
\hline
\end{tabular}

Note-SRT-F, fixed schedule of simple reaction time tasks; CRT-F, fixed schedule of two-choice reaction time tasks; SRT-R, random schedule of simple reaction time tasks; CRT-R, random schedule of two-choice reaction time tasks. 
Table 6

\begin{tabular}{|c|c|c|c|c|c|}
\hline \multirow[b]{2}{*}{ Task } & \multirow[b]{2}{*}{ Condition } & \multicolumn{2}{|c|}{ RT (msec) } & \multirow{2}{*}{$\begin{array}{c}\text { Anticipations/ } \\
\text { Second }\end{array}$} & \multirow{2}{*}{$\begin{array}{c}\text { Response Selection } \\
\text { Errors/Second }\end{array}$} \\
\hline & & Average & $S D$ & & \\
\hline \multirow[t]{2}{*}{ SRT-F } & Single task & 297 & 105 & 0.01 & \\
\hline & Dual task & 492 & 353 & 0.07 & \\
\hline \multirow[t]{2}{*}{ CRT-F } & Single task & 425 & 100 & 0.01 & 0.02 \\
\hline & Dual task & 624 & 294 & 0.02 & 0.04 \\
\hline
\end{tabular}

task conditions. In the first dual-task condition, the participants had to solve the simple products while concurrently performing an SRT-F task. This condition was compared with a dual-task condition in which the participants concurrently performed a CRT-F task. The data showed a significant effect of response selection with a moderate effect size. These results suggest that response selection is involved in solving simple arithmetical products. Just as in the previous two experiments, there was no significant load $\times$ problem size interaction.

In the next experiment, we tried to replicate this finding by comparing performance on the primary task while an SRT-R task was executed concurrently with a dual-task condition in which the secondary task was a CRT-R task.

\section{EXPERIMENT 4}

\section{Method}

Participants. Twenty first-year psychology students (16 of them female and 4 male) participated in the present experiment for course requirements and credit. The mean age was 20.2 years (range, 18.3-27.8 years). None of them had participated in the previous experiments.

Stimuli and Procedure. The stimuli and the procedure were the same as those in the previous three experiments. The only difference relates to the secondary tasks used. This experiment was based on the SRT-R task (see Experiment 1) and the CRT-R task (see Experiment 2).

\section{Results}

Latencies. Of the 13,440 experimental trials (672 products solved by 20 participants), 1,254 , or $9.3 \%$, were dropped due to coughing or voice key failure. As in the previous experiments, the eliminated trials were evenly distributed among the three conditions. Only the latencies of the correctly solved products were included in the analysis. A multivariate analysis based on the general linear model was conducted on the variable of load (three levels: control, SRT-R, or CRT-R). Planned comparisons showed that the difference between the control condition $(M=1,128 \mathrm{msec})$ and the SRT-R condition ( $M=1,261 \mathrm{msec}$ ) was significant $\left[F(1,19)=16.86, M S_{\mathrm{e}}=10,483.9, p<.001 ; d=.49\right]$. There was a significant dual-task coordination cost. Also, the difference between the SRT-R condition and the CRT-R condition $(M=1,394 \mathrm{msec})$ was significant $[F(1,19)=$ $10.74, M S_{\mathrm{e}}=16,505.0, p<.01 ; d=.39$ ]; the products were solved more quickly in the SRT-R condition than in the CRT-R condition.

The problem size $\times$ load interaction was investigated by analyzing the data by means of a 3 (load: control, SRT-R, or
CRT-R) $\times 2$ (problem size: small or large) within-subjects design. Mean latencies and standard deviations are displayed in Table 5. There were significant main effects of load $\left[F(2,38)=18.01, M S_{\mathrm{e}}=38,532, p<.001\right]$ and of problem size $\left[F(1,19)=133.83, M S_{\mathrm{e}}=48,833, p<.001\right]$. There was no significant load $\times$ problem size interaction $\left(F<1, M S_{\mathrm{e}}=16,734\right)$.

Accuracy. The accuracy was high. The difference between the control condition $(M=.96)$ and the CRT-R condition $(M=.94)$ was significant $\left[F(1,19)=5.24, M S_{\mathrm{e}}=\right.$ $0.0008, p<.05 ; d=.59$ ], as was the difference between the SRT-R condition $(M=.96)$ and the CRT-R condition $\left[F(1,19)=8.73, M S_{\mathrm{e}}=0.0003, p<.01 ; d=.57\right]$.

As in the previous experiments, an error analysis was conducted. This analysis showed that $87 \%$ of the errors were common table-related intrusions and that these intrusions were evenly distributed across conditions.

Secondary task performance. The data for the secondary tasks are displayed in Table 7. Just as in the previous experiments, performance on each secondary task was worse in the dual-task condition than in the single-task condition $\left[F(1,19)=222.09, M S_{\mathrm{e}}=1,456, p<.001\right.$, for the SRT-R task, and $F(1,19)=262.34, M S_{\mathrm{e}}=496, p<.001$, for the CRT-R task].

The correlations between primary task performance and each of the secondary tasks separately were not significant $(r=.15, p=.52$, in the SRT-R condition, and $r=.00$ in the CRT-R condition). As in the previous experiments, there were no trade-offs between primary task and secondary task performance.

\section{Discussion}

In this experiment, the role of response selection in solving simple products was studied by comparing performance under concurrent SRT-R and CRT-R conditions. The results showed a significant difference between the two dual-task conditions with a moderate effect size. Clearly, there was a significant effect of response selection. Also, in this experiment, there was no significant difference between small and large problems. The problem size $\times$ load interaction was not significant.

\section{ADDITIONAL ANALYSES}

Although the main focus of this article has been on the role of input monitoring and response selection in solving simple arithmetical products, the question arises as to how these two processes relate to each other. Are these processes independent of each other, or do they interact? 
Table 7

Experiment 4: Random Simple Reaction Time (SRT-R) and

\begin{tabular}{|c|c|c|c|c|c|c|}
\hline \multirow[b]{2}{*}{ Task } & \multirow[b]{2}{*}{ Condition } & \multicolumn{2}{|c|}{ RT (msec) } & \multirow{2}{*}{$\begin{array}{l}\text { Anticipations/ } \\
\text { Second }\end{array}$} & \multirow{2}{*}{$\begin{array}{l}\text { Response Selection } \\
\text { Errors/Second }\end{array}$} & \multirow[b]{2}{*}{ Correlatior } \\
\hline & & Average & $S D$ & & & \\
\hline \multirow[t]{2}{*}{ SRT-R } & Single task & 280 & 83 & 0.03 & & .95 \\
\hline & Dual $\mathrm{t}$ & 449 & 273 & 0.08 & & .89 \\
\hline \multirow[t]{2}{*}{ CRT-R } & Single task & 410 & 127 & 0.03 & 0.05 & .90 \\
\hline & Dual task & 522 & 269 & 0.07 & 0.10 & .90 \\
\hline
\end{tabular}

The purpose of this additional section is to investigate the relation between the two processes. The dependence/independence of the input monitoring and the response selection processes could be explored in two ways. First, the data from Experiments 1 and 2 were pooled and analyzed by means of a 2 (presentation schedule: fixed or random) $\times$ 2 (type of task: simple reaction or two-choice reaction) design, with repeated measures on the first factor. The main effect of presentation schedule was not significant $(F<1$, $d=.08)$. However, the main effect of type of task was significant $[F(1,38)=6.19, p<.05 ; d=.68]$. The presentation schedule $\times$ type of task interaction was not significant $(F<1, d=.55)$. Another way to study the dependence/ independence of these two processes is to look at the pooled data from Experiments 3 and 4. These data were analyzed by means of a similar 2 (presentation schedule) $\times 2$ (type of task) design, but now with repeated measures on the second factor. The main effect of type of task was significant $[F(1,38)=15.04, p<.001 ; d=.33]$, but the main effect of presentation schedule $(F<1, d=.28)$ and the presentation schedule $\times$ type of task interaction $(F<1, d=.17)$ were not. The fact that in both analyses, there was no significant presentation schedule $\times$ type of task interaction suggests that the two processes are probably independent of each other, at least in the context of simple mental arithmetic. It seems that the effects of input monitoring and response selection are additive and do not interact with each other.

\section{GENERAL DISCUSSION}

The goal of the present article was to study whether the executive processes of input monitoring and response selection are involved in solving simple mental arithmetical products. In an earlier study by Deschuyteneer and Vandierendonck (2005), the role of these two executive processes in the calculation of simple sums was investigated. Their results showed that response selection is important in solving simple sums, whereas input monitoring does not seem to be involved in solving such sums. Besides investigating whether these executive processes are involved in the calculation of simple products, the present study additionally allows us to examine the idea postulated by Deschuyteneer and Vandierendonck that response selection may be required during the retrieval of the correct answer from long-term memory. After all, simple multiplications are assumed to be solved by retrieving the answer from long-term memory (e.g., Ashcraft, 1992; Campbell, 1987, 1995; Campbell \& Graham, 1985; Collins \& Loftus, 1975; Dehaene \& Cohen,
1995; Galfano et al., 2004; Galfano et al., 2003; Geary et al., 1986; LeFevre et al., 1988; Miller et al., 1984; Rusconi et al., 2004; Siegler, 1988; Siegler \& Shrager, 1984; Thibodeau et al., 1996; Verguts \& Fias, 2005). Four experiments similar to those in the study of Deschuyteneer and Vandierendonck were conducted. The only difference was that, in the present study, the participants had to solve simple products, instead of simple sums, as a primary task.

The involvement of input monitoring was studied in Experiments 1 and 2 by comparing performance on the primary task (i.e., solving simple products) under concurrent fixed and random stimulus presentation conditions. Similarly, the role of response selection was investigated in Experiments 3 and 4 by comparing performance under concurrent simple reaction and two-choice reaction conditions. Our data showed no significant effect of input monitoring. This was the case in Experiments 1 and 2, and this remained so when the data from these two experiments were pooled in one analysis. Also, when the data from Experiments 3 and 4 were pooled, the effect of input monitoring was not significant. The estimated effect sizes were also very small. However, there was a significant effect of response selection in (1) Experiments 3 and 4, (2) the combined analysis based on these two experiments, and (3) the combined analysis based on the data from Experiments 1 and 2. All in all, the results were the same as those in the study of Deschuyteneer and Vandierendonck (2005). It seems that, just as for simple sums, the calculation of simple products is impaired by response selection and not by input monitoring.

The lack of an effect of input monitoring can be interpreted in two ways. Either input monitoring is not involved in solving simple arithmetical products, or this process does not entail executive control. The latter assumption, however, does not seem plausible. After all, Vandierendonck et al. (1998) found in their study that (1) a concurrent, randomly spaced simple reaction time task impairs supra span shortterm memory performance and (2) that at and below span level, the effect of input monitoring was significant in backward recall but not in forward. This finding, together with the fact that there was also no significant effect of input monitoring for the calculation of simple sums (Deschuyteneer \& Vandierendonck, 2005), makes the assumption more plausible that input monitoring is not involved in the solving of both simple arithmetical sums and products. However, this does not rule out the possibility that our temporal manipulation of this task characteristic was not adequate. Due to the fact that the products were presented at a slow rate, there was no need for the participants to control a sequence 
of events. Therefore, it could be interesting, in future research, to manipulate this task characteristic differently and to investigate whether this would lead to an effect of input monitoring. This could be done by varying the number of intervals at which a stimulus would be presented. For example, three intervals could be used. This would lead the participants to give more attention to the secondary task and might result in a higher load due to input monitoring. But then again, the present manipulation varied only temporal aspects of input monitoring, whereas it would certainly be interesting to study other aspects of attentional selectivity to environmental input. However, it is also possible that the null findings for input monitoring are due to the nature of the samples. Because the participants in the present study were well-educated adults, it could be that the attentional demands of solving simple multiplication problems decreased and, therefore, that the sensitivity of the present study to the influence of input monitoring was low.

Our results were similar to those in Deschuyteneer and Vandierendonck (2005) in that we found a significant but moderate effect of response selection. It seems that, just as for solving simple sums, response selection is involved in solving simple arithmetical products. Hence, this could lead to the assumption that response selection is involved in solving simple mental arithmetical sums and products (i.e., the so-called arithmetical facts). This finding confirms our hypothesis that if response selection is involved in the retrieval of arithmetical facts, it is involved in both the calculation of simple arithmetical sums and products. The possibility still exists, however, that response selection is not involved in the retrieval of the correct answer from long-term memory but that nonarithmetical processes, such as the encoding of the problem and the production of the answer, are impaired by response selection. For example, it has been suggested by previous studies that the encoding phase of stimuli may require response selection. Naveh-Benjamin, Craik, Perretta, and Tonev (2000) and Szmalec et al. (2005), for instance, found that concurrently executing a choice reaction time task in the stimulus presentation phase of a short-term memory task impairs recall. These findings seem to indicate that encoding may require response selection. So it could be that, just as in the memory task, some encoding operations require response selection in the multiplication task. There is, however, a difference between the encoding phase in a short-term memory task and the encoding phase while simple multiplications are solved. In memory tasks, not only are the stimuli encoded, but also the stimulus representations have to be maintained active in memory. Hence, two processes play a role in the stimulus presentation phase of a memory task - namely, encoding and maintenance processes. This is not the case in solving simple products. In our primary task, the products are solved trial by trial, and the arithmetical problems only have to be encoded and do not need to be maintained active across different trials. Thus, it could be that in the studies of Szmalec et al. and of Naveh-Benjamin et al., the effect of response selection occurred at the level of maintenance, rather than at the level of encoding. So the explanation that response selection is required in the encoding of arithmetical products remains speculative and is in need of further research.

Nevertheless, our findings constitute an additional support for the idea that response selection is involved in the retrieval of the correct solution from long-term memory. The data are consistent with the idea that the presentation of two numbers of an arithmetic problem results in the automatic activation of the number nodes in an interrelated network of associative links in long-term memory (e.g., Ashcraft, 1992; Campbell, 1987, 1995; Campbell \& Graham, 1985; Collins \& Loftus, 1975; Dehaene \& Cohen, 1995; Galfano et al., 2004; Galfano et al., 2003; Geary et al., 1986; Le-Fevre et al., 1988; Miller et al., 1984; Rusconi et al., 2004; Siegler, 1988; Siegler \& Shrager, 1984; Thibodeau et al., 1996; Verguts \& Fias, 2005). Activation then automatically spreads from the presented nodes along associative links to related number nodes, such as the sum or the product. After the automatic activation of possible responses, a response selection is required to complete the retrieval. As has already been mentioned in the introduction, findings from other sources are also consistent with the notion that a response selection occurs after the automatic activation of possible responses (e.g., Bunge et al., 2002; Lien \& Proctor, 2002).

As in previous studies (e.g., De Rammelaere et al., 2001; De Rammelaere \& Vandierendonck, 2001; Deschuyteneer \& Vandierendonck, 2005), none of the experiments showed a significant problem size $\times$ load interaction. If we make the assumption that a response selection is required, after the automatic activation of possible responses, to complete the retrieval, it may be that the duration of this response selection phase varies in such a way that some fact retrievals take more time than do others. This variation could be consistent with the size effect. However, the size effect is, in part, also caused by other factors, such as the amount of competition in the number network, the usage of more time-consuming strategies, and others. In other words, the degree of variation in response selection duration is expected to be much smaller than the size effect would suggest, and it is probably associated with individual differences (Barrouillet, Bernardin, \& Camos, 2004). According to this view, the size of the impairment that is due to secondary task response selection is expected to be more or less the same for any outcome of any multiplication. In other words, even if response selection duration did tend to increase with size, the problem size $\times$ load interaction would still be rather small. All these interpretations are, however, speculative and require further empirical and theoretical development.

To summarize, our results indicate that response selection is involved in the solving of simple arithmetical products, whereas input monitoring does not seem to be involved. So, when these results are combined with the results of Deschuyteneer and Vandierendonck (2005), the hypothesis that response selection is involved in solving arithmetical facts gains strength, whereas there is no support for the role of input monitoring.

However, the issue remains as to what is the origin of the observed dual-task interference with response selec- 
tion. The literature remains unclear with regard to whether interference with the process of response selection is due to a competition between the primary task and the secondary task for the same process (see the concept of structural bottleneck; Pashler, 1998) or to a shortfall in attentional resources when two attention-demanding tasks are performed simultaneously (cf. Barrouillet et al., 2004). Therefore, at this moment, we assign the observed interference with response selection to a competition for the same resources, just as for the process of input monitoring (see the introduction for a definition of input monitoring). After all, when the required amount of resources is large, two retrievals cannot be performed simultaneously, and this does not necessarily mean that there is a competition for the same process.

Furthermore, the question arises as to whether other components of executive functioning are involved in the calculation of simple mental arithmetical sums and products. Take, for example, the process of inhibition. Miyake et al. (2000) have already indicated, in their study, that inhibition may be a possible executive process (see also Baddeley, 1996). However, there are different types of inhibition. The one most clearly operationalized is interference control. Previous developmental studies concerning mental arithmetic have suggested that when a response needs to be selected from a range of automatically activated responses, the interference from incorrect responses needs to be controlled (e.g., Barrouillet, Fayol, \& Lathulière, 1997; Geary, Hamson, \& Hoard, 2000). An analysis of the errors in these studies showed that most errors were common table-related intrusions, which is in keeping with the idea that an interference control component may be involved in solving such simple arithmetical sums and products. An analysis of the errors in the present experiments also showed that most errors were common table-related intrusions. Also, studies from totally different fields have suggested that when a response needs to be selected from a range of automatically activated responses, the incorrect responses need to be inhibited (e.g., Allain, Carbonnell, Burle, Hasbroucq, \& Vidal, 2004; Conway \& Engle, 1994; Kane \& Engle, 2003). Therefore, in the future, it would be interesting to investigate whether such an interference control component is involved when the retrieval strategy is used to come to the correct solution of an arithmetic problem. So, besides the process of response selection, which is needed to select the correct response from a range of automatically activated responses, an interference control component may also be involved that inhibits the incorrect responses. But other components, such as memory updating, also may play a role. Since memory updating is important in short-term memory tasks (see Szmalec \& Vandierendonck, 2006), it may be that memory updating is involved also in the calculation of simple arithmetic problems.

Finally, it would also be interesting to examine other arithmetical operations, such as subtraction and division. In fact, previous research indicates that these types of arithmetic problems are not solved only by retrieving the correct answer from memory (e.g., Campbell, 1999a;
Dehaene \& Cohen, 1997; Geary, Frensch, \& Wiley, 1993; Mauro, LeFevre, \& Morris, 2003). For the domain of simple subtraction, for example, it seems that several backup strategies, such as counting and reference to a related addition problem, may be used to solve simple subtractions (Carpenter \& Moser, 1984; Seyler, Kirk, \& Ashcraft, 2003; Siegler, 1989; Suppes, Hyman, \& Jerman, 1967; Svenson \& Hedenborg, 1979; Woods, Resnick, \& Groen, 1975). Therefore, it can be interesting to study whether response selection and input monitoring are involved in the calculation of simple subtractions and/or divisions. It would, for example, be interesting to study whether response selection is also involved in these arithmetical operations. Or is response selection involved only in solving simple sums and products? If simple subtractions and divisions are solved by using other strategies than just retrieving the answer from memory, it can be expected that the use of more complex strategies to solve such arithmetic problems may entail that participants perform not just one retrieval, but multiple retrievals, to solve such a problem. Consequently, if response selection is involved in retrieving the correct answer from long-term memory, a complexity by load interaction may be expected if multiple retrievals have to be executed to solve simple subtractions and divisions. And what about input monitoring? Thus far, it seems that input monitoring does not impair the calculation of simple arithmetic problems. But if there are other strategies besides the retrieval of the correct answer from long-term memory used to solve such problems, it could be important to investigate whether input monitoring is involved in the calculation of such arithmetic problems and, if so, whether it is involved in the same way.

\section{REFERENCES}

Allain, S., Carbonnell, L., Burle, B., HasbroucQ, T., \& Vidal, F. (2004). On-line executive control: An electromyographic study. Psychophysiology, 41, 113-116.

AsHCRAFT, M. H. (1982). The development of mental arithmetic: A chronometric approach. Developmental Review, 3, 213-236.

AshCraft, M. H. (1992). Cognitive arithmetic: A review of data and theory. Cognition, 44, 76-106.

BADDELEY, A. D. (1986). Working memory. Oxford: Oxford University Press.

BADDELEY, A. D. (1990). Human memory: Theory and practice. Hove, U.K.: Erlbaum.

BAdDELEy, A. D. (1996). Exploring the central executive. Quarterly Journal of Experimental Psychology, 49A, 5-28.

BADDELEY, A. D. (2000). The episodic buffer: A new component of working memory? Trends in Cognitive Sciences, 4, 417-423.

Baddeley, A. D., \& Hitch, G. J. (1974). Working memory. In G. H. Bower (Ed.), The psychology of learning and motivation (Vol. 8, pp. 4789). New York: Academic Press.

BAROODY, A. J. (1985). Mastery of the basic number combinations: Internalization of relationships or facts? Journal for Research in Mathematics Education, 16, 83-98.

Barrouillet, P., Bernardin, S., \& CAmos, V. (2004). Time constraints and resource sharing in adults' working memory spans. Journal of Experimental Psychology: General, 133, 83-100.

Barrouillet, P., Fayol, M., \& Lathulière, E. (1997). Selecting between competitors in multiplication tasks: An explanation of the errors produced by adolescents with learning difficulties. International Journal of Behavioral Development, 21, 253-275.

Blankenberger, S. (2001). The arithmetic tie effect is mainly encodingbased. Cognition, 82, B15-B24. 
Bunge, S. A., Hazeltine, E., Scanlon, M. D., Rosen, A. C., \& GabriELI, J. D. E. (2002). Dissociable contributions of prefrontal and parietal cortices to response selection. NeuroImage, 17, 1562-1571.

CAmpBell, J. I. D. (1987). Network interference and mental multiplication. Journal of Experimental Psychology: Learning, Memory, \& Cognition, 13, 109-123.

CAMPBELl, J. I. D. (1995). Mechanisms of simple addition and multiplication: A modified network interference theory and simulation. Mathematical Cognition, 1, 121-164.

Campbell, J. I. D. (1997). On the relation between skilled performance of simple division and multiplication. Journal of Experimental Psychology: Learning, Memory, \& Cognition, 23, 1140-1159.

Campbell, J. I. D. (1999a). Division by multiplication. Memory \& Cognition, 27, 791-802.

Campbell, J. I. D. (1999b). The surface form $\times$ problem size interaction in cognitive arithmetic: Evidence against an encoding locus. Cognition, 70, 25-33.

Campbell, J. I. D., \& Graham, D. J. (1985). Mental multiplication skill: Structure, process, and acquisition. Canadian Journal of Psychology, 39, 338-366.

Campbell, J. I. D., \& Gunter, R. (2002). Calculation, culture, and the repeated operand effect. Cognition, 86, 71-96.

Campbell, J. I. D., Kanz, C. L., \& Xue, Q. (1999). Number processing in Chinese-English bilinguals. Mathematical Cognition, 5, 1-39.

Carpenter, T. P., \& Moser, J. M. (1984). The acquisition of addition and subtraction concepts in grades one through three. Journal for Research in Mathematics Education, 15, 179-202.

Chochon, F., Cohen, L., van de Moortele, P. F., \& Dehaene, S. (1999). Differential contributions of the left and right inferior parietal lobules to number processing. Journal of Cognitive Neuroscience, 11, 617-630

CoHen, J. (1988). Statistical power analysis for the behavioral sciences (2nd ed.). Hillsdale, NJ: Erlbaum.

Collins, A. M., \& Loftus, E. F. (1975). A spreading activation theory of semantic processing. Psychological Review, 82, 407-428.

Conway, A. R. A., \& Engle, R. W. (1994). Working memory and retrieval: A resource-dependent inhibition model. Journal of Experimental Psychology: General, 4, 354-373.

Dehaene, S., \& Cohen, L. (1995). Towards an anatomical and functional model of number processing. Mathematical Cognition, 1, 83120.

Dehaene, S., \& Cohen, L. (1997). Cerebral pathways for calculation: Double dissociation between rote verbal and quantitative knowledge of arithmetic. Cortex, 33, 219-250.

De Rammelaere, S., Stuyven, E., \& Vandierendonck, A. (1999). The contribution of working memory resources in the verification of simple mental arithmetic sums. Psychological Research, 62, 72-77.

De Rammelaere, S., Stuyven, E., \& Vandierendonck, A. (2001). Verifying simple arithmetic sums and products: Are the phonological loop and the central executive involved? Memory \& Cognition, 29, 267-273

De Rammelaere, S., \& Vandierendonck, A. (2001). Are executive processes used to solve simple mental arithmetic production tasks? Current Psychology Letters: Behaviour, Brain \& Cognition, 2, 79-89.

Deschuyteneer, M., \& VandierendoncK, A. (2005). Are "input monitoring" and "response selection" involved in solving simple mental arithmetical sums? European Journal of Cognitive Psychology, 17, 347-370.

DeStefano, D., \& LeFevre, J.-A. (2004). The role of working memory in mental arithmetic. European Journal of Cognitive Psychology, 16, 353-386.

Fürst, A. J., \& Hitch, G. J. (2000). Separate roles for executive and phonological components of working memory in mental arithmetic. Memory \& Cognition, 28, 774-782.

Galfano, G., Mazza, V., Angrilli, A., \& Umiltà, C. (2004). Electrophysiological correlates of stimulus-driven multiplication facts retrieval. Neuropsychologia, 42, 1370-1382.

Galfano, G., Rusconi, E., \& Umiltà, C. (2003). Automatic activation of multiplication facts: Evidence from the nodes adjacent to the product. Quarterly Journal of Experimental Psychology, 56A, 31-61.

Geary, D. C., Frensch, P. A., \& Wiley, J. G. (1993). Simple and complex mental subtraction: Strategy choice and speed-of-processing differences in younger and older adults. Psychology \& Aging, 8, 242-256.
Geary, D. C., Hamson, C. O., \& Hoard, M. K. (2000). Numerical and arithmetical cognition: A longitudinal study of process and concept deficits in children with learning disability. Journal of Experimental Child Psychology, 77, 236-263.

Geary, D. C., Widaman, K. F., \& Little, T. D. (1986). Cognitive addition and multiplication: Evidence for a single memory network. Memory \& Cognition, 14, 478-487.

HiтcH, G. J. (1978). The role of short-term working memory in mental arithmetic. Cognitive Psychology, 10, 302-323.

JIANG, Y., \& KANWISHER, N. (2003a). Common neural mechanisms for response selection and perceptual processing. Journal of Cognitive Neuroscience, 15, 1095-1110.

JiAnG, Y., \& KANWISHER, N. (2003b). Common neural substrates for response selection across modalities and mapping paradigms. Journal of Cognitive Neuroscience, 15, 1080-1094.

KANE, M. J., \& ENGLE, R. W. (2003). Working-memory capacity and the control of attention: The contributions of goal neglect, response competition, and task set to Stroop interference. Journal of Experimental Psychology: General, 132, 47-70.

LeFevre, J.-A., Bisanz, J., \& Mrkonjic, L. (1988). Cognitive arithmetic: Evidence for obligatory activation of arithmetic facts. Memory \& Cognition, 16, 45-53.

Lemaire, P., ABDi, H., \& FAYol, M. (1996). The role of working memory resources in simple cognitive arithmetic. European Journal of Cognitive Psychology, 8, 73-103.

Lien, M.-C., \& Proctor, R. W. (2002). Stimulus-response compatibility and psychological refractory period effects: Implications for response selection. Psychonomic Bulletin \& Review, 9, 212-238.

Logie, R. H., Gilhooly, K. J., \& Wynn, V. (1994). Counting on working memory in arithmetic problem solving. Memory \& Cognition, 22, 395-410.

Mauro, D. G., LeFevre, J.-A., \& Morris, J. (2003). Effects of problem format on division and multiplication performance: Division facts are mediated via multiplication-based representations. Journal of Experimental Psychology: Learning, Memory, \& Cognition, 29, 163-170.

Miller, K. F., Perlmutter, M., \& Keating, D. (1984). Cognitive arithmetic: Comparison of operations. Journal of Experimental Psychology: Learning, Memory, \& Cognition, 10, 46-60.

Miyake, A., Friedman, N. P., Emerson, M. J., Witzki, A. H., HowERTER, A., \& WAGER, T. D. (2000). The unity and diversity of executive functions and their contributions to complex "frontal lobe" tasks: A latent variable analysis. Cognitive Psychology, 41, 49-100.

Naveh-Benjamin, M., Craik, F. I. M., Perretta, J. G., \& Tonev, S. T. (2000). The effects of divided attention on encoding and retrieval processes: The resiliency of retrieval processes. Quarterly Journal of Experimental Psychology, 53A, 609-625.

Nö̈L, M.-P., Désert, M., Aubrun, A., \& Seron, X. (2001). Involvement of short-term memory in complex mental calculation. Memory \& Cognition, 29, 34-42.

PAshler, H. (1994). Dual-task interference in simple tasks: Data and theory. Psychological Bulletin, 116, 220-244.

PAshler, H. (1998). The psychology of attention. Cambridge, MA: MIT Press.

Pesenti, M., Thioux, M., Seron, X., \& De Volder, A. (2000). Neuroanatomical substrates of Arabic number processing, numerical comparison, and simple addition: A PET study. Journal of Cognitive Neuroscience, 12, 461-479.

Rickard, T. C., Romero, S. G., Basso, G., Wharton, C., Flitman, S., \& Grafman, J. (2000). The calculating brain: An fMRI study. Neuropsychologia, 38, 325-335.

Rusconi, E., Galfano, G., Speriani, V., \& Umiltà, C. (2004). Capacity and contextual constraints on product activation: Evidence from task-irrelevant fact retrieval. Quarterly Journal of Experimental Psychology, 57A, 1485-1511.

SCHubert, T. (1999). Processing differences between simple and choice reactions affect bottleneck localization in overlapping tasks. Journal of Experimental Psychology: Human Perception \& Performance, 2, 408-425.

Schumacher, E. H., Elston, P. A., \& D’Esposito, M. (2003). Neural evidence for representation-specific response selection. Journal of Cognitive Neuroscience, 15, 1111-1121.

Seitz, K., \& Schumann-Hengsteler, R. (2000). Mental multiplica- 
tion and working memory. European Journal of Cognitive Psychology, 12, 552-570.

Seitz, K., \& Schumann-Hengsteler, R. (2002). Phonological loop and central executive processes in mental addition and multiplication. Psychologische Beiträge, 44, 275-302.

Seyler, D. J., Kirk, E. P., \& AshCRAft, M. H. (2003). Elementary subtraction. Journal of Experimental Psychology: Learning, Memory, \& Cognition, 29, 1339-1352.

SIEGLER, R. S. (1988). Strategy choice procedures and the development of multiplication skill. Journal of Experimental Psychology: General, 117, 258-275.

SiEgLER, R. S. (1989). Hazards of mental chronometry: An example from children's subtraction. Journal of Educational Psychology, 81, 497-506.

Siegler, R. S., \& Shrager, J. (1984). A model of strategy choice. In C. Sophian (Ed.), Origins of cognitive skills (pp. 229-293). Hillsdale, NJ: Erlbaum.

SupPes, P., Hyman, L., \& JeRman, M. (1967). Linear structural models for response and latency performance in arithmetic on computer-controlled terminals. In J. P. Hill (Ed.), Minnesota symposia on child psychology (Vol. 1, pp. 160-200). Minneapolis: University of Minnesota Press.

Svenson, O., \& Hedenborg, M. L. (1979). Strategies used by children when solving simple subtractions. Acta Psychologica, 43, 477-489.

Szmalec, A., \& VAndierendoncK, A. (2006). Estimating the executive demands of a one-back choice RT task by means of the selective interference paradigm. Manuscript under revision.
Szmalec, A., Vandierendonck, A., \& Kemps, E. (2005). Response selection involves executive control: Evidence from the selective interference paradigm. Memory \& Cognition, 33, 531-541.

Thibodeau, M. H., LeFevre, J.-A., \& Bisanz, J. (1996). The extension of the interference effect to multiplication. Canadian Journal of Experimental Psychology, 50, 393-396.

VANDIERENDONCK, A. (2000a). Bias and processing capacity in the generation of random time intervals. Cognitive Science Quarterly, 2, 205-233.

VANDIERENDONCK, A. (2000b). Is judgment of random time intervals biased and capacity-limited? Psychological Research, 63, 199-209.

Vandierendonck, A., De Vooght, G., \& VAn der Goten, K. (1998). Interfering with the central executive by means of a random interval repetition task. Quarterly Journal of Experimental Psychology, 51A, 197-218.

VERGUTS, T., \& FIAS, W. (2005). Interacting neighbors: A connectionist model of retrieval in single-digit multiplication. Memory \& Cognition, 33, 1-16.

Woods, S. S., Resnick, L. B., \& Groen, G. J. (1975). Experimental tests of five process models for subtraction. Journal of Educational Psychology, 67, 17-21.

(Manuscript received May 19, 2004; accepted for publication December 30, 2004.) 